University of Nebraska - Lincoln

DigitalCommons@University of Nebraska - Lincoln

2013

\title{
Increases in Cognitive and Linguistic Processing Primarily Account for Increases in Speaking Rate With Age
}

Ignatius S. B. Nip

San Diego State University, inip@mail.sdsu.edu

Jordan R. Green

University of Nebraska-Lincoln, jgreen4@unl.edu

Follow this and additional works at: https://digitalcommons.unl.edu/specedfacpub

Nip, Ignatius S. B. and Green, Jordan R., "Increases in Cognitive and Linguistic Processing Primarily Account for Increases in Speaking Rate With Age" (2013). Special Education and Communication Disorders Faculty Publications. 81.

https://digitalcommons.unl.edu/specedfacpub/81

This Article is brought to you for free and open access by the Department of Special Education and Communication Disorders at DigitalCommons@University of Nebraska - Lincoln. It has been accepted for inclusion in Special Education and Communication Disorders Faculty Publications by an authorized administrator of DigitalCommons@University of Nebraska - Lincoln. 


\title{
Increases in Cognitive and Linguistic Processing Primarily Account for Increases in Speaking Rate With Age
}

\author{
Ignatius S. B. Nip, San Diego State University \\ Jordan R. Green, University of Nebraska-Lincoln \\ Corresponding author - Ignatius Nip, School of Speech, Language, and Hearing Sciences, \\ San Diego State University, 5500 Campanile Dr., San Diego, CA 92182-1518; email inip@mail.sdsu.edu
}

\begin{abstract}
Age-related increases of speaking rate are not fully understood, but have been attributed to gains in biologic factors and learned skills that support speech production. This study investigated developmental changes in speaking rate and articulatory kinematics of participants aged $4(N=7), 7(N=10), 10(N=9), 13(N=7), 16(N=9)$ years, and young adults $(N=11)$ in speaking tasks varying in task demands. Speaking rate increased with age, with decreases in pauses and articulator displacements but not increases in articulator movement speed. Movement speed did not appear to constrain the speaking. Rather, age-related increases in speaking rate are due to gains in cognitive and linguistic processing and speech motor control.
\end{abstract}

$\mathrm{T}$ he rate at which we speak provides information to others about our gender, dialect, cognition, health, and age. For instance, young children and even adolescents talk at a slower rate than adults (Amster, 1984; Haselager, Slis, \& Rietveld, 1991; Kowal, O'Connell, \& Sabin, 1975; Sturm \& Seery, 2007; Walker, Archibald, Cherniak, \& Fish, 1992). The prolonged development of speaking rate is particularly surprising given that adolescents have already established the rules and sounds of their language (Kowal et al., 1975). The factors that allow for increased rates of speech with age are not fully understood, but have been attributed to gains in biologic factors (e.g., anatomic growth, neurologic and neuromuscular maturation) and learned skills that support rapid spoken language production (motor learning; semantic, lexical, and phonologic access; and motor programming and planning). At the level of speech motor control, talkers have only three options to increase their rate of speech: (a) decrease the extent of oral movement, (b) increase the speed of oral movement, or (c) decrease the number and duration of pauses (Campbell \& Dollaghan, 1995). Investigating how these variables and speaking rate covary with age will have implications for understanding the contributions of biologic and spoken language processing factors on speaking rate development.

\section{Evidence for Constraining Biologic Factors}

One untested biologic hypothesis is that the slowed rate of speech in children is, in part, due to relatively slow central and peripheral neural conduction speeds. Findings from studies on limbs using transcranial magnetic stimulation suggest that conduction times in children are limited by the fastest corticomotoneuronal efferent nerves (Müller \& Hömberg, 1992) with central conduction times decreasing significantly with age (Müller, Hömberg, \& Lenard, 1991). The conduction velocities of neural pathways innervating orofacial structures may also increase with age. Indirect evidence comes from a small study that demonstrates that the latency time of the perioral reflex of adults are faster than those of children (Barlow, Finan, Bradford, \& Andreatta, 1993). Support for a limiting role of movement speed on the development of speaking rate would be provided by the observation of a strong association (i.e., a correlation of greater than 0.8, as defined by Cohen, 1988) between age-related changes in movement speed and speaking 
rate. More specifically, if speed is observed to be slow in children across both very simple and complex speaking tasks, this finding would suggest that the changes in movement speed are predominantly biologically rather than linguistically driven.

\section{Evidence of Motor Learning}

Immature motor control is characterized by inefficiencies in motor control (Goldfield, Kay, \& Warren, 1993). For example, in early reaching, poor motor control results in an overshoot of the displacement of the arm and hand (Jeannerod, 1988) and poor force regulation results in excessive pincer force during grasping (Potter, Kent, Lindstrom, \& Lazarus, 2006). Poor position control in early speech development may similarly result in articulatory target overshoot, specifically, excessively large articulatory displacements (Green \& Nip, 2010). Immature control of the speech motor system has been observed in young children, who produce larger and slower articulator movements than adults do (Goffman \& Smith, 1999; Green \& Wilson, 2006; Riely \& Smith, 2003; Smith \& Gartenberg, 1984; Smith \& Goffman, 1998; Smith \& Zelaznik, 2004; Walsh \& Smith, 2002). The relatively large oral excursions produced by children may account for the slower rate of speech, particularly if they are not accompanied by proportional increases in movement speed. This finding would provide support for the suggestion that an essential process in the development of speaking rate is the optimal tuning of the speech motor control system through motor learning.

\section{Evidence for a Reduced Capacity to Formulate Spoken Language}

Speech motor control, including speed of articulator movement and consequently speech, is also dependent on cognitive, linguistic, and motor workloads (Green \& Nip, 2010). The processing demands on spoken language production that are imposed by different speaking tasks may vary depending on factors including attention, utterance familiarity (word frequency and phonotactic probability), utterance length, and syntactic complexity. For example, the slowing of speech during the performance of a manual visuomotor tracking task is evidence of the influence of attentional demands on speech motor control (Dromey \& Benson, 2003). In addition, children speak faster during simple speaking tasks, such as the repetitions of simple syllables, than during more demanding speaking tasks, such as conversational speech (Haselager et al., 1991). The relations between speaking rate and task demands suggest that children speak slower than adults, in part, because their articulator movement speeds are slowed by their reduced capacity to formulate spoken language. Therefore, the influence of speaking task demands on children's rate of speech would be supported by the observation that children's speeds of articulator movement are faster for low-demand speaking tasks, but slower for high-demand speaking tasks across development.

Another way to determine the contribution of limited spoken language processing on the development of speaking rate is to examine age-related changes in pausing patterns. Prior research has shown that children gradually reduce the number and length of pauses, a change that has been attributed to developmental gains in cognitive and linguistic processing (Kowal et al., 1975). A talker pauses more frequently and for longer as speaking tasks become more cognitively and linguistically demanding (Greene, 1984; Greene \& Cappella, 1986). For example, adults pause longer, thereby decreasing their speaking rate, while speaking on a topic spontaneously than while speaking on the same topic after preparing an outline (Mitchell, Hoit, \& Watson, 1996); similarly, young children speak more slowly in spontaneous speech as compared to familiar utterances (Walker \& Archibald, 2006). The observation that changes in pausing, rather than in articulator movements, primarily account for age-related changes in speaking rate would suggest that gains in spoken language processing play a more prominent role in the development of speaking rate than do biologic factors.

\section{Research Questions}

The current study investigated age-related changes in speaking rate, articulatory kinematics (i.e., displacements and speeds), and pause duration across several speaking tasks that varied from simple alternating oral movements to story retell. The stimuli were designed to elicit different levels of processing demands (motor, linguistic, or cognitive processing) on spoken language production. Our central research question was to determine whether changes in speaking rate are primarily due to the constraining effects of biologic factors (movement speed), motor learning (refinement of displacements) or due to gains in spoken language processing (interactions between age, task demands, speaking rate, and pausing patterns). Evidence for biologic constraints on the development of speaking rate would be supported by two possible findings: (a) the observations that the speed of speech movements 
are not significantly different across all ages and tasks (which vary in processing demands), or (b) age-related changes in movement speed are not significantly different across tasks. In either scenario, the common course of movement changes cannot be attributed to differences across tasks, but instead to underlying biologic factors. Evidence for motor learning factors would be supported if oral excursions decrease with age and do not change with task demands. In contrast, observations of task-specific changes in movement speed (i.e., speed varies predictably across tasks) would provide evidence that spoken language processing (i.e., motor, linguistic, and cognitive) may drive developmental changes in speaking rate. For example, we might anticipate that the movement speed for simple syllables will change little with age, whereas movement speed would increase significantly for more challenging speaking tasks. The observation that articulatory displacements become smaller as speaking rate increases with age would provide support for the suggestion that an essential process in the development of speaking rate is the optimal tuning of the speech motor control system through motor learning. Finally, a complementary analysis was performed on connected speech samples at each age to further discern the relative contributions of biologic, motor learning, and spoken language production processing changes on the development of speaking rate. The suggestion that speaking rate increases are primarily due to increases in spoken language production processing would be supported by observations of a significantly greater change in pause duration than articulatory kinematics or a disassociation between age-related changes in speaking rate and articulatory kinematics.

\section{Method}

\section{Participants}

Data were collected on a total of 78 participants; however, 24 participants were not included in the analysis because they were either unable to complete the tasks, were unwilling to have the markers placed on their faces, or were identified as having a speech or language delay during the speech and language screening. As a result, 54 participants in the following age groups are included in this report: 4- (5 males, 2 females, $M=4.45$ years, $S D=0.39$ years $), 7-(5$ males, 5 females, $M=7.57, S D=0.29$ years $), 10$ - (5 females, 4 males, $M=10.40$ years, $S D=0.27$ years $), 13$ - ( $3 \mathrm{fe}-$ males, 4 males, $M=13.31$ years, $S D=0.27$ years), and 16-year-olds ( 5 females, 4 males, $M=16.39$ years, $S D=$ 0.32 years) and adults ( 5 males, 6 females, $M=23.75$ years, $S D=3.52$ years). All participants lived in Nebraska, spoke American English with a standard Midwest dialect as their first language, and were primarily from middle-class backgrounds. The majority of participants identified as White, not Hispanic. Four participants identified as White and Hispanic. Four participants did not identify their race as White. One identified as African American, not Hispanic; the second identified as Native American and White, not Hispanic; another identified as White, African American, and Hispanic, and the last identified as White, African American, Native American, and White, not Hispanic. None of the participants had any history of speech, language, hearing, or learning difficulties. Each participant passed a hearing screening at 1,2 , and $4 \mathrm{kHz}$ at $20 \mathrm{~dB}$ HL for children and at $25 \mathrm{~dB}$ HL for adults, following American Speech-Language-Hearing Association (ASHA) guidelines for audiological screening (ASHA Panel on Audiologic Assessment, 1997). Child participants also passed a receptive and expressive language screening; 4-year-olds were screened using the Core Language subtests (Sentence Structure, Word Structure, Expressive Vocabulary subtests) of the Clinical Evaluation of Language Fundamentals-Preschool (CELF-P) 2nd edition (Wiig, Secord, \& Semel, 2004). The language skills of the 7-, 10-, 13-, and 16-year-olds were screened using the Clinical Evaluation of Language Fundamentals-4 (CELF-4) Screening test (Semel, Wiig, \& Secord, 2004). Based on an informal conversational speech sample, a certified speech-language pathologist did not identify and articulation delays or disorders.

\section{Data Collection}

Lip and jaw movements were captured using a threedimensional motion capture system (Motion Analysis, Ltd., Santa Rosa, CA). This optically based system has eight infrared cameras (Eagle) with 1.3 megapixel resolution that were calibrated to the manufacturer's specifications prior to each data collection session. The system used a sampling rate of 120 frames per second for each camera. Fifteen spherical reflective markers, approximately $2 \mathrm{~mm}$ in diameter, were placed on the forehead, eyebrows, nose, lips and jaw with double-sided hypoallergenic adhesive tape as shown in Figure 1. A rigid plate with a miniature microphone and four markers was also attached to each subject's forehead. The forehead marker array was used for the off-line subtraction of head movements from those of the lower 
lip marker. High-resolution digital video and audio (44.1 kHz and 16 bits) signals of the participants were also recorded and used for parsing of bilabial opening and for acoustic analyses, as described below.

Participants were seated in front of the camera system. Video clips providing instructions and demonstrations of the speech tasks were projected on a large screen in front of the participant. The video clips ensured that the instructions (detailed below under "Speaking Tasks") and demonstrations were presented in an identical manner and uniform rate to each participant. For younger participants, an investigator also sat in front of the child in order to keep him or her facing the cameras. Occasional coaching on tasks was provided, particularly for younger children.

All movement traces were low-pass filtered at $7 \mathrm{~Hz}$ using a Butterworth filter (Butterworth, 1930). To obtain lip and jaw movements, the 3D Euclidean distance between the lower lip marker and the head marker at the right top head was calculated for each task. This calculation effectively subtracted head movement from the movement of the lower lip marker.

The initial opening gesture of the lower lip marker for a voiced bilabial segment was examined in order to compare the same movement gesture across all tasks. Specifically, the syllable of / b/ followed by an open

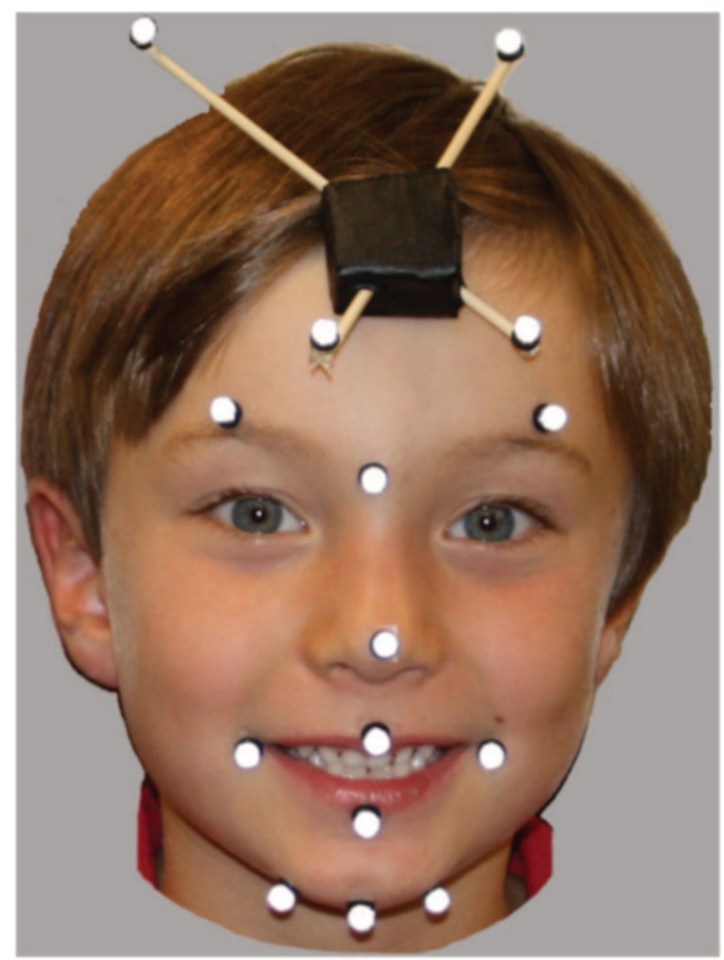

Figure 1. Marker set used for articulatory movement recording. vowel was parsed from the narrative retell ("Bobby"), sentence repetition ("buy Bobby"), syllable repetition ("uhba"), and diadochokinetic or diadochokinetic (DDK) rates ("buh"). These syllables were then measured using custom algorithms developed for Matlab. Zero-crossings in velocity signals were used to detect the onset and offset of each opening gesture associated with each vowel. Displacement $(\mathrm{mm})$ was also measured and was defined as the difference between the position of the lower lip marker at bilabial closure and at full opening for the vowel. Measures of peak speed $(\mathrm{mm} / \mathrm{s})$ and duration (s) were also obtained.

\section{Speaking Tasks}

All speaking tasks were purposefully loaded with bilabial segments to engage use of the lips and jaw. The large number of voiced segments allowed a Matlabbased algorithm (Green, Beukelman, \& Ball, 2004) to automatically identify pauses in the speech signals. Four different speaking tasks were used to vary the amount of cognitive, linguistic, and motor processing demands. Speaking tasks included a narrative retell task using the "Bats, Boots, and Beets" story (Green, Nip, Wilson, Mefferd, \& Yunusova, 2010), sentence repetition ("Buy Bobby a puppy"), syllable repetition ("uhba"), and a DDK task. For the narrative retell, participants were shown a picture corresponding to each sentence of the story as the story was read to them. They were then asked to retell the story while viewing the same pictures. During the DDK task, participants were asked to repeat "buh" fast and clearly and to do as many repetitions as possible in a single breath. For all tasks, speakers were asked to produce speech at their habitual rate and loudness.

The DDK, a speech-like task used by speech-language clinicians to examine the fastest alternating motion rates in the oromotor system, had the fewest task demands (Ziegler, 2002). Therefore, this task was used to identify the maximum repetition rate during a task that was relatively unencumbered by cognitive or linguistic processing demands or by motor planning (Haselager et al., 1991), providing a measure of the capacity of the participant's speech motor system (Ziegler, 2002). Relative to the DDK task, the syllable task was thought to increase linguistic processing demands because it imposed a syllabic structure (i.e., a vowel-consonant-vowel [VCV] sequence that conforms to English phonology rather than a continuous string of $\mathrm{CV}$ sequences) on the phonemic sequence. To further increase cognitive and linguistic demands, the simple sentence task was used to impose 
semantic and syntactic structure. Finally the narrative retell task was considered to be the most complex as it required participants to remember the story, in addition to producing a sequence of sentences in a simple narrative structure.

\section{Analyses}

All speech samples were orthographically transcribed and speaking rates were calculated for each task in syllables per minute. The rate in which speech is produced has been measured in several different ways in previous studies. In the fluency literature, speaking rate is commonly defined as the number of fluent and disfluent syllables divided by the amount of time to produce that utterance (e.g., Hall, Amir, \& Yairi, 1999; Kelly \& Conture, 1992). In contrast, articulation rate of the same utterance is the number of syllables divided by time during fluent speech (e.g., Kelly \& Conture, 1992; Ryan, 2000). Studies in speaking rate of typically developing children have typically measured speaking rate as the number of syllables or words produced divided by the total time, including pausing, needed to produce that utterance (e.g., Flipsen, 2002, 2003). Articulation rate in these studies typically refer to the number of syllables or words produced divided by the duration, with pausing time removed (e.g., Flipsen, 2002, 2003; Walker \& Archibald, 2006). As all the speakers in the current study were typically-developing individuals with no history of speech or language difficulties, speaking rate was operationally defined as the amount of time to produce an utterance, including pause times; articulation rate was operationally defined as the amount of time to produce an utterance with any pausing removed. The percentage of time for pausing measured in order to examine processing time a participant needed to generate an utterance.

Custom Matlab algorithms were used to determine the total pause time, defined as silence for $100 \mathrm{~ms}$ or longer but are not stop gaps (Green et al., 2004), for all speech tasks. For each audio file, an amplitude threshold (Green et al., 2004) was specified to allow the algorithm to determine the minimum acoustic energy needed to distinguish the speech signal from noise. Occasionally, in less than approximately 10 occasions, stop gaps (or silences) for the production of /b/ were identified as being pauses. These were discarded from the final analysis as it did not represent linguistic and cognitive processing. Previous studies (e.g., Haselager et al., 1991) have shown that decreases in pausing time may account for some of the developmental changes in speaking rate. No pausing was found for the DDK, syllable, and sentence task; only the narrative retell was found to contain interutterance pauses. This finding was anticipated and suggested that the DDK, syllable, and sentence task did not require either a respiratory or cognitive pause even in the 4-year-old children. Therefore, only the narrative retell task was analyzed for pausing time.

\section{Statistical Analyses}

For each participant, means of the peak speeds, displacement, and duration were measured across the multiple repetitions of each speaking task for the lower lip marker. The means for peak speed, displacement, and duration were Winsorized using the 25th and 75th percentile Tukey hinges to calculate the upper and lower bounds of the distribution for a speaking task for each age. Observations of peak speed, displacement, and duration that fell outside the calculated upper and lower bounds were replaced with the calculated maximal or minimal values.

Bivariate correlations between age, sex, speaking rate, peak speed, duration, and displacement for the DDK, syllable repetition, and sentence repetition tasks were conducted. Bivariate correlations using the same variables and percent pausing time were also conducted for the narrative retell task. Statistical analyses tested for the effects of age and speaking task (DDK, syllable, sentence, narrative) on speaking rate, peak speed, duration, and displacement. A multivariate analysis of variance (MANOVA) was used to determine if age and task cause significant changes in speaking rate, peak speed, displacement, and duration. Separate two-way univariate analyses of variance were then used determine significant differences in speaking rate, peak speed, duration, and displacement between age groups and tasks.

\section{Results}

Bivariate correlations between age, sex, speaking rate, peak speed, duration, and displacement for each of the speaking tasks are shown in Tables 1-4. Table 1 presents these correlations for the DDK task, Table 2 for the syllable repetition task, and Table 3 for the sentence repetition task. Percent pausing time, considered to be an indicator of cognitive, linguistic, and motor processing, 
Table I. Correlations Between Age, Sex, Speaking Rate, Peak Speed, Duration, and Displacement for the Diadochokinetic Task

\begin{tabular}{llllll}
\hline & Sex & $\begin{array}{l}\text { Speaking } \\
\text { rate }\end{array}$ & $\begin{array}{l}\text { Peak } \\
\text { speed }\end{array}$ & $\begin{array}{l}\text { Dura- } \\
\text { tion }\end{array}$ & $\begin{array}{l}\text { Displace- } \\
\text { ment }\end{array}$ \\
\hline Age & .09 & $.75^{* * *}$ & .16 & $.69 * * *$ & -.05 \\
Sex & & .11 & -.07 & -.11 & -.13 \\
Speaking rate & & -.13 & $-.95^{* * *}$ & $-.39 *$ \\
Peak speed & & & .11 & $.95 * * *$ \\
Duration & & & & $.38 *$ \\
\hline
\end{tabular}

$* p<.05 ; * * * p<.001$

Table 2. Correlations Between Age, Sex, Speaking Rate, Peak Speed, Duration, and Displacement for the Syllable Repetition Task

\begin{tabular}{|c|c|c|c|c|c|}
\hline & Sex & $\begin{array}{l}\text { Speaking } \\
\text { rate }\end{array}$ & $\begin{array}{l}\text { Peak } \\
\text { speed }\end{array}$ & $\begin{array}{l}\text { Dura- } \\
\text { tion }\end{array}$ & $\begin{array}{l}\text { Displace- } \\
\text { ment }\end{array}$ \\
\hline Age & .11 & -.01 & $.40 *$ & $-.29 *$ & .20 \\
\hline Sex & & .15 & .06 & .07 & .05 \\
\hline Speaking rate & & & -.10 & $-.28 *$ & -.15 \\
\hline Peak speed & & & & .21 & $.95 * *$ \\
\hline Duration & & & & & $.46 * *$ \\
\hline
\end{tabular}

$* p<.05 ; * * p<.01$

Table 3. Correlations Between Age, Sex, Speaking Rate, Peak Speed, Duration, and Displacement for the Sentence Repetition Task

\begin{tabular}{|c|c|c|c|c|c|}
\hline & Sex & $\begin{array}{l}\text { Speaking } \\
\text { rate }\end{array}$ & $\begin{array}{l}\text { Peak } \\
\text { speed }\end{array}$ & $\begin{array}{l}\text { Dura- } \\
\text { tion }\end{array}$ & $\begin{array}{l}\text { Displace- } \\
\text { ment }\end{array}$ \\
\hline Age & . 11 & $.43^{* *}$ & $.50 * *$ & $-.32 *$ & $.34 *$ \\
\hline Sex & & .01 & .02 & -.02 & .03 \\
\hline Speaking rate & & & .16 & $-.5 \mathrm{I} * *$ & -.06 \\
\hline Peak speed & & & & -.12 & $.90 * *$ \\
\hline Duration & & & & & $.28 *$ \\
\hline
\end{tabular}

$* p<.05 ; * * p<.01$

was calculated only during the narrative task, as the other tasks were all repetition tasks with no inherent interword pauses. Correlations between age, sex, speaking rate, peak speed, duration, displacement, and percent pausing time for the narrative retell task are shown in Table 4. Because sex was not found to be significantly correlated with any variable, it was removed from further analyses.

A MANOVA was conducted to examine the multivariate difference by age and task on the dependent variables speaking rate, articulation rate, peak speed, displacement, and duration. A significant Age $\times$ Task interaction was found, Wilks $=.005, F=7661.61, p<.001$.
Both the main effect of age, Wilks $=.332, F=9.48$, $p<.001$, and task, Wilks $=.102, F=43.67, p<.001$, were significant. Follow-up analyses of variance for each dependent variable are reported below.

\section{Age and Task Effects on Speaking Rate and Articula- tion Rate}

Speaking rate and articulation rate for each task and age is shown in Figure 2. A between-groups factorial analysis of variance with follow-up analyses using the least significant difference (LSD) minimum mean difference procedure was performed to examine the effects of age and task on speaking rate. A significant main effect of age $F(5,188)=20.81, p<.001$ was found, with speaking rate increasing with age up until 13 years of age. The main effect of task was also statistically significant $F(3$, $188)=108.04, p<.001$; however, these main effects were qualified by a significant interaction of task and age, $F(15,188)=3.20, p<.001$.

The LSD minimum mean difference was used to follow up the Age $\times$ Task interaction. The 4-, 10-, 13-yearolds demonstrated the same pattern; narratives had significantly slower speaking rates than the other tasks and the DDK task had significantly higher speaking rates than all the other tasks. For the 7-year-olds, narratives similarly had slower speaking rates than the other tasks. In addition sentences were also found to have significantly lower speaking rates than DDK and syllables. The 16-year-olds also demonstrated significantly higher speaking rates for DDK than the other tasks and sentences had significantly higher speaking rates than narratives. Adults were similar to 16-yearolds; they demonstrated significantly higher speaking rates for DDK than the other task but sentences had higher speaking rates than both syllables and narratives.

Next, in examining age differences within a task, For the DDK task, 4-year-olds were shown to significantly lower speaking rates than did the other age groups, 7 -year-olds had significantly lower speaking rates than did 13-year-olds, and adults had significantly higher speaking rates for every age group except the 13-yearolds. For the syllable task, 7-year-olds had significantly higher speaking rates than did 4-, 10-, 16-year-olds, and adults; 13-year-olds had significantly higher speaking rates than did 4- and 10-year-olds. The sentence repetition task demonstrated that 13-year-olds had significantly higher speaking rates for 4-, 7-, and 10-yearolds; 16-year-olds had significantly higher speaking rates than 4-year-olds; and adults had significantly 
Table 4. Correlations Between Age, Sex, Speaking Rate, Peak Speed, Duration, Displacement, and Percent Pausing Time for the Narrative Task

\begin{tabular}{|c|c|c|c|c|c|c|}
\hline & Sex & Speaking rate & Peak speed & Duration & Displacement & Pct. pausing \\
\hline Sex & & -.01 & -.14 & -.09 & -.18 & -.06 \\
\hline Speaking rate & & & $.33^{*}$ & $-.67 * *$ & .04 & $-.67 * *$ \\
\hline Peak speed & & & & $-.44 *$ & $.89 * *$ & $-.37 *$ \\
\hline Duration & & & & & -.03 & $.43^{* *}$ \\
\hline
\end{tabular}

$* p<.05 ; * * p<.01$

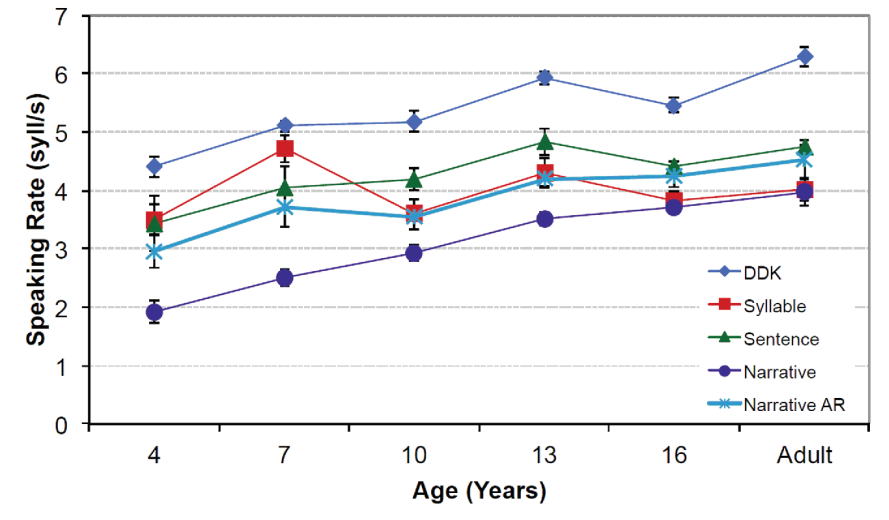

Figure 2. Speaking rate in syll/s for diadochokinetic (DDK), syllable repetition, sentence repetition, and narrative retell and articulation rate (AR) in syll/s for narrative retell. ARs for diadochokinetic, syllable repetition and sentence repetition tasks were the same as the speaking rates.

higher speaking rates than did 4- and 7-year-olds. Finally, the narrative task revealed that 10 -year-olds had significantly higher speaking rates than did 4-year-olds, 13-year-olds had significantly higher speaking rates than did 4- and 7-year-olds, and 16-year-olds and adults both had significantly higher speaking rates than did 10-, 7-, and 4-year-olds. Taken together, speaking rate increases with increased age and with decreased language formulation demands.

The articulation rate of the narratives was compared with the speaking rates of the other tasks. Because the other tasks were found to have no pausing, the speaking rates for the DDK, syllable, and sentence repetitions are also the articulation rates. Similar to speaking rate, a between-groups factorial analysis of variance with followup analyses using the LSD minimum mean difference procedure was conducted to examine the effects of age and task on articulation rate. A main effect of age, $F(5$,
$188)=14.88, p<.001$, was found with articulation rate increasing with age. The main effect of task was significant, $F(3,188)=48.90, p<.001$. Similar to speaking rate, these main effects were qualified by a significant interaction of task and age, $F(15,188)=1.90, p<.05$.

Follow-up analyses using the LSD minimum mean difference were used to further examine the Age $\times$ Task interaction. For 4-, 10-, 13-, 16-year-olds, and adults, the DDK task had significantly higher articulation rates than the other tasks. Additionally, 13-year-olds demonstrated significantly higher articulation rates for sentences than narratives, and adults demonstrated significantly higher articulation rates for syllables than sentences. For the 7-year-olds, the DDK and syllable repetition task had significantly higher articulation rates than both sentences and narratives.

Focusing on age differences within a task, for the DDK task, adults and 13-year-olds had higher articulation rates than all the other groups. In addition, 4-yearolds had significantly lower articulation rates than 16-, 10-, and 7-year-olds. Syllable repetitions for 7 year-olds were significantly higher in articulation rates than 4-, 10-, 16-year-olds, and adults, and significantly higher for 13-year-olds than 4- and 10-year-olds. For the sentence repetition task, 13-year-olds and adults had significantly higher articulation rates than 4- and 7-year-olds. Similarly 10- and 16-year-olds had significantly higher articulation rates than 4-year-olds. Finally, for the narrative retell task, 16-year-olds and adults had significantly higher articulation rates for 4-, 7-, and 10-year-olds; 7and 13-year-olds also had significantly higher articulation rates than 4-year-olds. Similar to the speaking rate results, articulation rate also increases with increased age and with decreased language formulation demands.

The change in percent pausing time for narrative retells across age groups is shown in Figure 3. A one-way analysis of variance was conducted to examine the effect of age on percent pausing time. A main effect of age, $F(5,48)=9.84, p<.001$, was found. Post hoc tests using the LSD procedure using an alpha level of .05 were con- 
ducted. The 4- and 7-year-olds had significantly greater percent pausing time than all the other age groups except 10-year-olds. No significant differences were found between 10-, 13-, 16-year-olds, and adults.

\section{Age and Task Effects on Peak Speed}

The peak lower lip movement speeds for each speaking task are shown in Figure 4. A between-groups factorial analysis of variance with follow-up analyses was performed to examine the effects of age and task on peak speed. There was no significant interaction between age and task. A main effect of age, $F(5,188)=17.66, p<.001$, was found. There was also a main effect of task, $F(3,188)$ $=17.06, p<.001$, with faster speeds for tasks requiring more spoken language production processing demands.

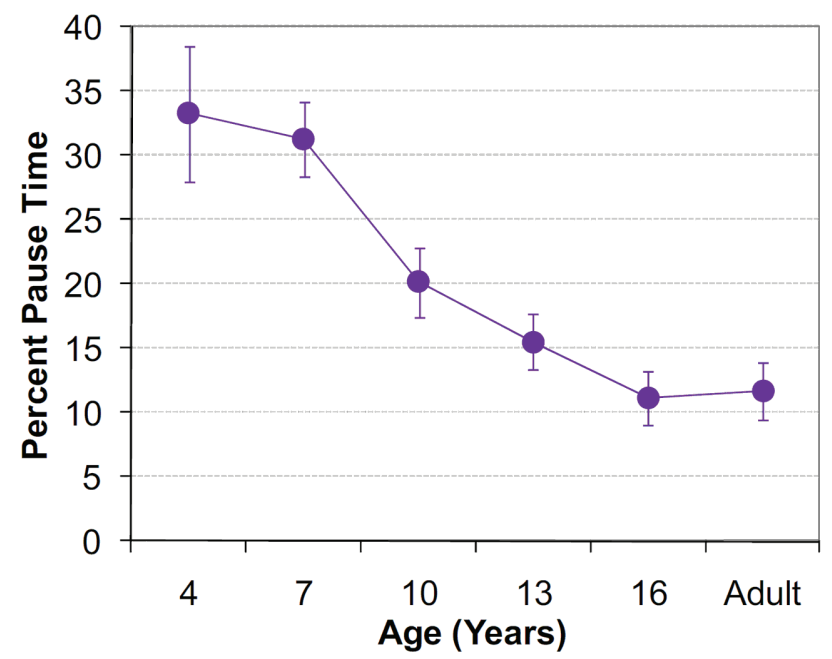

Figure 3. Percent pause time for narrative retell task.

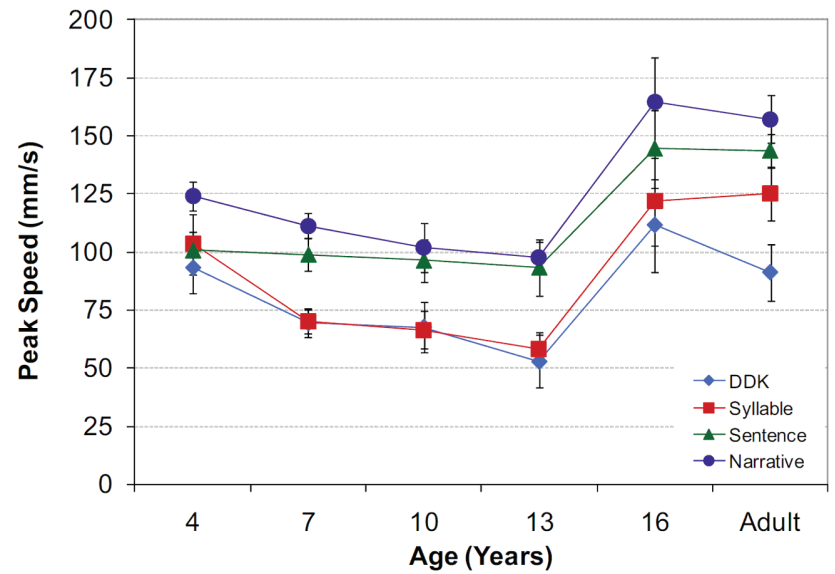

Figure 4. Peak speed of the lower lip marker in $\mathrm{mm} / \mathrm{s}$ for the diadochokinetic (DDK), syllable repetition, sentence repetition, and narrative retell tasks.
Post hoc tests using the LSD procedure with an alpha level of .05 were performed. Across tasks, 4-year-olds were significantly faster than 13 -year-olds. The 16-yearolds were significantly faster than were 4-, 7-, 10-, and 13-year-olds. Adults had significantly faster peak speeds than did 7-, 10-, and 13-year-olds. Across all ages, DDK and syllable repetition tasks were produced with significantly slower peak speeds than the sentence repetition and narrative retell tasks. Overall, 16-year-olds and adults had the fastest peak speeds. Across all participants, tasks requiring greater spoken language production processing demands were produced with faster peak speeds than those requiring less spoken language production processing demands.

\section{Age and Task Effects on Duration}

Duration of the bilabial opening for each speaking task is shown in Figure 5. A between-groups analysis of variance was also conducted on duration. A significant interaction between age and task was also found, $F(15,188)=2.12, p<.01$, primarily because the narratives had the longest durations except for the 16-yearolds and adult groups for whom syllable repetition had longer durations. Significant main effects for both age, $F(5,188)=18.76, p<.001$, and task, $F(3,188)=105.06$, $p<.001$, were found as well.

Post hoc tests using the LSD minimum mean difference were used to determine how cell means differed from each other. The 4-year-olds had significantly shorter durations for DDK than the other tasks, and sen-

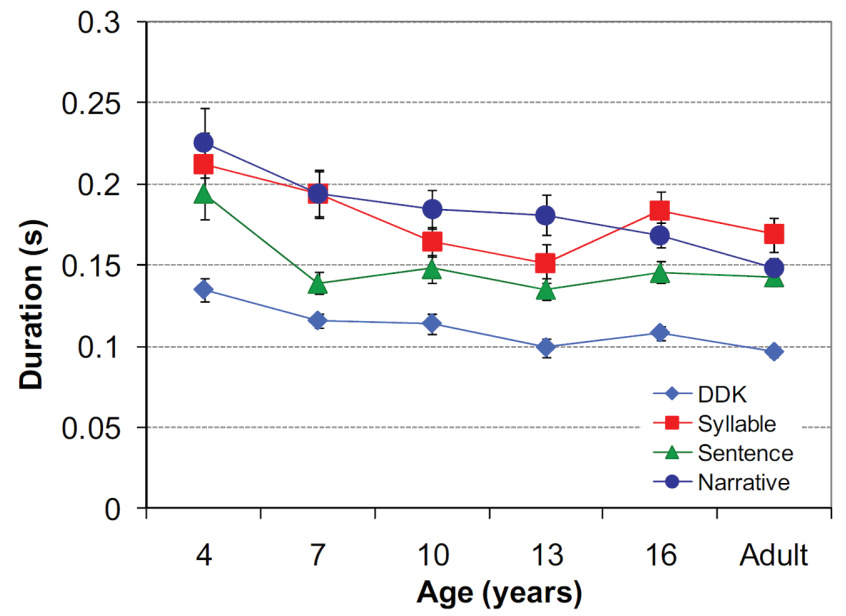

Figure 5. Duration of bilabial opening in seconds for the diadochokinetic (DDK), syllable repetition, sentence repetition, and narrative retell tasks. 
tences had significantly shorter durations than narratives. The 7-year-olds had significantly longer durations for both sentences and DDK when compared to syllables or narratives. The 10-year-olds had significantly shorter durations for DDK than for the other tasks, and narratives showed significantly longer durations than the other tasks. Thirteen-year-olds also had shorter durations for DDK than the other tasks. In addition, narratives had significantly longer durations than syllables or sentences for this age group. Finally, both the 16-yearolds and adults had significantly shorter durations for DDK than other tasks and sentences had significantly shorter durations than syllables.

The LSD minimum mean difference was also used to determine how age groups differed in their duration for each task. In the DDK tasks, 4-year-olds had significantly longer durations than did 10-, 13-, 16-year-olds, and adults. In the syllable repetition task, 4-year-olds had significantly longer durations than all groups except for 7-year-olds, who had significantly longer durations than did 10- or 13-year-olds. In addition, 16-year-olds had longer durations than did 13-year-olds in this task. For the sentence repetition task and the narrative task, 4-year-olds had significantly longer durations than did the other age groups. For narratives, adults had significantly shorter durations than did 7-, 10-, and 13-year-olds. Overall, durations also increased with increased processing for spoken language production and durations generally decreased with age.

\section{Age and Task Effects on Displacement}

The displacement of bilabial opening for each speaking task is shown in Figure 6. A between-groups analysis of variance was also conducted on displacement. No significant interaction was found. The main effects of age, $F(5,188)=15.14, p<.001$, and task, $F(3,188)=46.56$, $p<.001$, were significant.

Post hoc tests using the LSD procedure with an alpha level of .05 were performed to examine the main effect of age. Four-year-olds had significantly larger displacements than did the 7-, 10-, 13-year-olds. The 7-yearolds had smaller displacements than did 16-year-olds but larger displacement than did 13-year-olds. Both 10and 13-year-olds had smaller displacements than did 16 -year-olds and adults. Overall, the youngest participants (4-year-olds) and the oldest participants (16-yearolds and adults) produced larger displacements for bilabial opening than did 7-, 10-, and 13-year-olds.

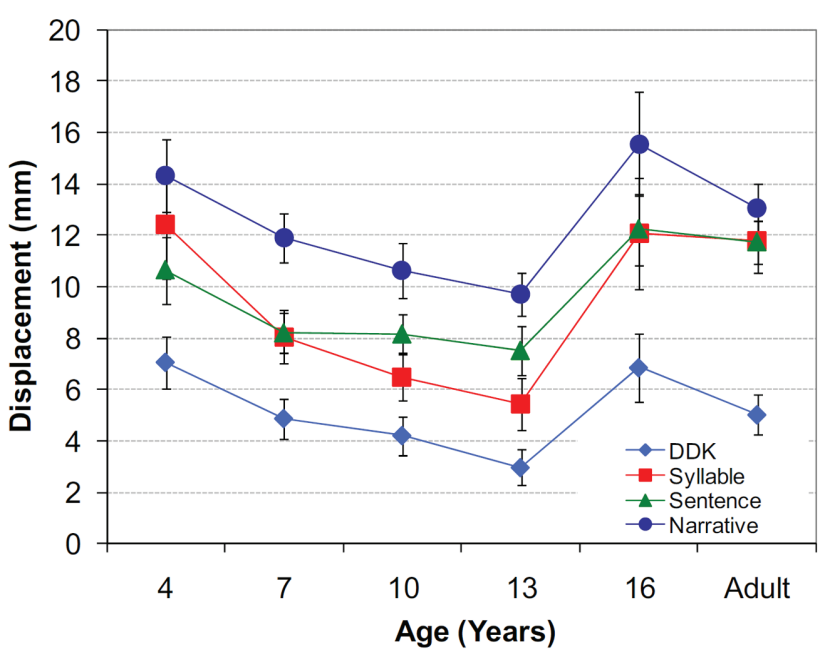

Figure 6. Displacement of the lower lip marker for bilabial opening in millimeters for the diadochokinetic (DDK), syllable repetition, sentence repetition, and narrative retell tasks.

Post hoc tests using the LSD procedure using an alpha level of .05 were performed to examine the main effect of task. For all age groups the DDK task had significantly smaller displacements as compared to other tasks. Both the syllable repetition and sentence repetition tasks had significantly smaller displacements as compared to the narrative retell task. There was no significant difference in displacement between the syllable and sentence repetition tasks. Generally, displacements increased with tasks requiring greater spoken language processing.

\section{Discussion}

The current study investigated developmental changes in speaking rate and articulatory kinematics in speaking tasks varying in task complexity. The data suggest that speaking rate increases gradually with age; however, movement speed and displacement decrease from preschool to 13 years of age before sharply increasing. These findings suggest that the development of speaking rate can be characterized by two major phases. The first phase occurred between 4 and 13 years when the greatest increases in speaking rate occurred. This change was clearly driven by decreases in movement displacement for the DDK, syllable, and sentence tasks and by decreases in movement displacement and pause time for the narrative task, and not increases in movement speed. The second phase occurred between 13 and 16 years of age 
when increases in speaking rate were primarily driven by increases in both peak speed and displacement with concomitant decreases in pauses time. The observation of gradual increases in speaking rate across the ages was striking given that it occurred in the context of more abrupt, underlying changes in speech motor control, specifically, the nonmonotonic increases in peak speed and displacement. One interpretation of these findings is that they demonstrate how, over time, children adapt to significant changes in their vocal tract anatomy to make gradual progress toward achieving adult-like speech performance. Taken together, the findings also suggest that increases in speaking rate are achieved through efficiencies in motor control and processing that support spoken language production. In contrast, evidence for the contribution of biologically-driven aspects (i.e., movement speed) was minimal with one exception being the major shift in articulatory kinematics that occurred between 13 and 16 years of age, which is during a period of accelerated vocal tract growth.

All of the variables measured in this study were affected by task demands; however, the demands of spoken language production produced opposite effects on speaking rate and articulatory kinematic variables. Specifically, across all age groups, speaking rate was faster for tasks with less spoken language production demands, such as DDK, and slower for tasks with greater spoken language production demands, such as sentences and narrative retell. Peak speed, duration, and displacement showed the opposite trend, with less demanding tasks produced with slower peak speeds, shorter durations, and smaller displacements than were more demanding tasks. One interpretation of these findings is that in young talkers' articulator movements become less efficient as the demands for spoken language increase.

\section{Developmental Trends}

Speaking and articulation rate changes with age. Speaking and articulation rate for all speaking tasks increased gradually from 4 to 13 years of age, replicating previous findings in the literature (e.g., Amster, 1984; Haselager et al., 1991; Kowal et al., 1975; Walker et al., 1992). Speaking rate did not change after 13 years of age, replicating earlier findings that 12-year-old children speak at approximately $90 \%$ of the adult speaking rate (Walsh \& Smith, 2002). However, a nonsignificant decrease in speaking rate was observed between 13 and 16 years of age for the DDK, syllable repetition, and sentence repetition tasks. This nonmonotonic trend in speaking rate is similar to previously reported findings that developmental changes in speaking rate are not necessarily linear. For example, Kowal et al. (1975) found that although 14- and 18-year-olds had very similar speaking rates, a transient increase in speaking rate was observed between the ages of 14 and 16 years. In addition, earlier studies of speaking rate have observed a plateau in speaking rate between the ages of 3 and 5 years (Pindzola, Jenkins, \& Lokken, 1989) and a transient decrease in speaking rate at the age of 5 years (Walker \& Archibald, 2006). In the current investigation, the percentage of pausing time during narrative retell decreased with age. This finding is similar to previous findings that demonstrate the percentage of pausing decreased from the ages of 4 to 16 years (Haselager et al., 1991; Kowal et al., 1975).

The duration of the speech movements, or the time it took for participants to produce a bilabial opening, indicated the net effect of the age-related changes observed in articulatory speed and displacement. Duration steadily decreased with age, but was significantly longer for 4- and 7-year-olds than it was for the older groups. Duration decreased as speaking rates increased. This finding is similar to those reported in earlier studies demonstrating that sentence durations decrease from 7 years of age to adults, suggesting that speech segments are produced with shorter durations with age (Chermak \& Schneiderman, 1985). This decrease in pausing and duration may represent a decrease in the time needed to: (a) conceptualize and formulate an utterance (Kowal et al., 1975; Rochester, 1973) and (b) motorically plan an utterance as increased motor practice has been shown to decrease planning time (Oytam, Neilson, \& O'Dwyer, 2005).

\section{Biologic Factors Did Not Have an Obvious Influence on the Development of Speaking Rate}

Unlike previous studies, this investigation examined how speaking rate development changes in the context of underlying articulatory kinematics. Although speaking rate increased incrementally between 4 and 13 years of age, the kinematic measures show a very different developmental course. Peak speed of articulatory movement decreased from 4 to 13 years before dramatically increasing between the ages of 13 to 16 years, demonstrating a strong disassociation between speaking rate and peak speed development. This finding suggests that peak speed may play only a minor role in limiting the rate at which children speak. 
Age-related changes in movement displacement closely parallel changes in movement speed. Generally, 4-year-olds were not significantly different in displacements as compared to the older participants (16-year-olds and adults) despite their smaller anatomical structures; this finding replicates earlier studies that demonstrate 5-year-olds do not have smaller displacements than adults during speech (Riely \& Smith, 2003). However, Riely and Smith (2003) only examined two age groups: 5 -year-olds and adults; the current study examined age groups between 4 years and adulthood. In the current investigation, displacement significantly decreased from the ages of 4-7 years of age, then plateaued between 7 and 13 years of age, before increasing again at 16 years of age. The similar displacements among the 7-, 10-, and 13-year-olds suggest that the ability to scale oral movements has a protracted developmental course. Potentially, this may reflect refinement in the scaling of articulatory displacements, similar to findings in the limb literature for grasping (e.g., Potter et al., 2006). Another possible reason for some of the age-related changes in the displacements may be anatomical growth. Previous research has demonstrated a major growth spurt in the vocal tract between the ages of 13 and 16 years (Fitch \& Giedd, 1999; Vorperian et al., 2009) and may explain the large increase in articulatory displacements found between these ages.

\section{Task Effects}

Speaking rate decreased with increasing production demands. Across all age groups, speaking rate slowed predictably in response to increasing task demands. In the current study, the DDKs, which had the least demands on cognitive, linguistic, and motor processing, were produced with faster speaking rates than were the sentence repetitions. Sentences, which were presumed to impose the greater spoken language production processing demands than DDK but less than the narratives, were produced with faster speaking rates than were the narrative retell tasks. Similar task effects on speaking rate have been reported in prior research on speech development. For instance, DDK speaking rates have been found to be significantly faster than spontaneous speech across childhood (Haselager et al., 1991). In addition, speaking material that is highly familiar, such nursery rhymes, and therefore presumed to require less cognition or language formulation resources, are produced at significantly faster speaking rates than less familiar sentences in preschoolers (Walker \& Archibald, 2006).
Adults show similar trends in speaking rate changes with speaking task; speaking tasks that are perceived as more difficult are associated with slower speaking rates, primarily due to more frequent and longer pauses (Mitchell et al., 1996).

Speaking rates for the syllable repetition task were less predictable than were speaking rates observed for the other tasks. Specifically, the syllable repetition task was presumed to have required more processing than the DDK task but less than the sentence repetition task. Therefore, the rate of speech during this task was predicted to fall between the DDK and sentence repetition tasks. In the current study, the speaking rate for the syllable repetition decreased with age, in relation to the other tasks. For instance, syllables are produced quite quickly in relation to the other tasks for younger participants but older participants, such as the 16-year-olds and the adults produced them at the same rate as the narrative retell. One interpretation of this finding is that the younger participants processed the syllables like a nonlinguistic task such as DDK, whereas adults processed the syllables more like meaningful speech. The reason for this putative change in processing is not obvious but could be related to changes in how speech representations are affected by emerging semantic, lexical, phonologic, and even literacy skills (Munson, Swenson, \& Manthei, 2005; Stoel-Gammon, 2011).

Peak speed, duration, and displacement. A consistent task effect was also seen in the peak articulatory speed data. In contrast to the speaking rate data, less demanding speaking tasks (i.e., DDK and syllable repetition) were produced with significantly slower movement speeds than were more demanding speaking tasks (i.e., sentences and narratives). This somewhat paradoxical result (speed might be expected to be faster during low-demand task than during high-demand task) suggests that factors other than processing demands govern the speed at which talkers move their articulators. One possible explanation for this finding is that lip displacements were larger for the high-demand tasks than for the low-demand tasks. It has long been known that large displacements are produced at faster speed than small displacements (see Ostry, Keller, \& Parush, 1983). The observation that lip displacements become larger during the more complex tasks suggests that articulator movements become less efficient as the demands for spoken language increase. An alternative explanation is that the more demanding tasks were more distinctly or hyper-articulated (Lindblom, 1990) because they contained linguistically relevant information. In 
contrast, talkers may have hypoarticulated the simple stimuli (i.e., truncate displacement) because they were willing to underspecify speech sounds because the goal of the task was to produce the syllables as rapidly as possible without having to convey linguistic information. Prior research has consistently demonstrated that articulatory displacements are truncated during rapidly produced syllables or speech (Mefferd \& Green, 2010; Westbury \& Dembowski, 1993). In short, if the achievement of adult-like speaking rates primary involves decreasing articulatory displacements, it is not surprising to see that articulatory movement speeds decrease proportionately.

The task-related findings on movement speed are also consistent with Lindblom's (1990) concept of listener-oriented articulatory control, where the specification of articulatory movements varies depending on the speaker's perception of the listeners' demands. Thus, for the sentence repetition and the narrative retell tasks, talkers may have increased the extent of their articulatory movements to enhance speech clarity for the purpose of conveying linguistic information.

Similarly, task effects were observed in the duration of lip and jaw opening, with duration generally decreasing with decreasing processing demands. Surprisingly, the older participants (16-year-olds and adults) produced syllables with longer durations than the narratives. Previous research has demonstrated that a shift in motor planning may occur as children increase cognitive and linguistic processing. Older children and adults decrease the duration of some phonemes and syllables when a simple (SVO) sentence is embedded within a complex sentence, similar to adult speakers; however, younger children do not show any differences in segmental duration between simple and complex sentences (Sadagopan \& Smith, 2008). Sadagopan and Smith (2008) suggest that this change in duration may be the result of increased linguistic maturity and flexibility that allows older talkers to plan longer sequences at a time rather than using word-by-word or syllable-by-syllable strategy. This hypothesis may account for the difference in task effects for the 16-year-olds and the adults as compared to the other age groups. The older participants have the flexibility to decrease the segmental duration for the $/ b /$ in order to produce longer utterances in the narrative retell; however, longer durations are utilized for shorter utterances such as simple syllables, perhaps to allow for greater accuracy in producing the phonetic targets. Similarly, the hypothesis put forth by Sadagopan and Smith (2008) may also account for the high degree of similarity of the durations for bilabial openings for the syllable, sentence, and narrative retell task of the younger group because this group lacks the ability to motorically plan units larger than a syllable or a word.

\section{Conclusion}

The current investigation was designed to test several hypotheses regarding the roles of biologic factors, and motor learning and spoken language processing gains on the typical development of speaking rate. Speaking rate increased between 4 and 13 years of age and consistent task effects were seen across all ages. More complex tasks were produced at slower speaking rates than the less complex tasks. Pausing data from the narratives suggest that children become more efficient in cognitive, linguistic, and motor planning. Consistent task effects were also seen for kinematic measures; however, the direction of the task effects for speaking rate and articulatory speed were in the opposite directions; less complex tasks had faster speaking rates but slower peak speeds as compared to more complex tasks. This effect may represent talkers' attempts to meet the demands of each task in the most efficient manner.

\section{References}

American Speech-Language-Hearing Association Panel on Audiologic Assessment. (1997). Guidelines for audiologic screening. Rockville, MD: American Speech-LanguageHearing Association.

Amster, B. J. (1984). The rate of speech of normal preschool children (Articulatory development, fluency, language, stuttering). Unpublished doctoral dissertation, Temple University, Philadelphia, PA.

Barlow, S. M., Finan, D. S., Bradford, P. T., \& Andreatta, R. D. (1993). Transitional properties of the mechanically evoked perioral reflex from infancy through adulthood. Brain and Language, 623, 181-188.

Butterworth, S. (1930). On the theory of filter amplifiers. Experimental Wireless and the Wireless Engineer, 7, 536- 541.

Campbell, T. F., \& Dollaghan, C. A. (1995). Speaking rate, articulatory speed, and linguistic processing in children and adolescents with severe traumatic brain injury. Journal of Speech and Hearing Research, 38, 864-875.

Chermak, G. D., \& Schneiderman, C. R. (1985). Speech timing variability of children and adults. Journal of Phonetics, 13, $477-480$.

Cohen, J. (1988). Statistical power analysis for the behavioral sciences (2nd ed.). Hillsdale, NJ: Erlbaum. 
Dromey, C., \& Benson, A. (2003). Effects of concurrent motor, linguistic, or cognitive tasks on speech motor performance. Journal of Speech, Language and Hearing Research, 46, 1234-1246.

Fitch, W. T., \& Giedd, J. (1999). Morphology and development of the human vocal tract: A study using magnetic resonance imaging. Journal of Acoustic Society of America, 106, 1511-1522.

Flipsen, P., Jr. (2002). Longitudinal changes in articulation rate and phonetic phrase length in children with speech delay. Journal of Speech, Language and Hearing Research, 45, 100-110.

Flipsen, P., Jr. (2003). Articulation rate and speech-sound normalization failure. Journal of Speech, Language and Hearing Research, 46, 724-737.

Goffman, L., \& Smith, A. (1999). Development and phonetic differentiation of speech movement patterns. Journal of Experimental Psychology: Human Perception and Performance, 25, 649-660.

Goldfield, E. C., Kay, B. A., \& Warren, W. H. (1993). Infant bouncing: The assembly and tuning of action systems. Child Development, 64, 1128-1142.

Green, J. R., Beukelman, D. R., \& Ball, L. J. (2004). Algorithmic estimation of pauses in extended speech samples of dysarthric and typical speech. Journal of Medical Speech-Language Pathology, 12, 149-154.

Green, J. R., \& Nip, I. S. B. (2010). Some organization principles in early speech development. In B. Maassen \& P. H. H. M. Van Lieshout (eds.), Speech motor control: New developments in basic and applied research (pp. 171-188). Oxford, UK: Oxford University Press.

Green, J. R., Nip, I. S. B., Wilson, E. M., Mefferd, A. S., \& Yunusova, Y. (2010). Lip movement exaggerations during infant directed speech. Journal of Speech, Language, and Hearing Research, 53, 1529-1542.

Green, J. R., \& Wilson, E. M. (2006). Spontaneous facial motility in infants: A 3D kinematic analysis. Developmental Psychobiology, 48, 16-28.

Greene, J. O. (1984). Speech preparation processes and verbal fluency. Human Communication Research, 11, 61-84. doi: 10.1111/j.1468-2958.1984.tb00038.x

Greene, J. O., \& Cappella, J. N. (1986). Cognition and talk: The relationship of semantic units to temporal patterns of fluency in spontaneous speech. Language and Speech, 29, 141-157.

Hall, K. D., Amir, O., \& Yairi, E. (1999). A longitudinal investigation of speaking rate in preschool children who stutter. Journal of Speech, Language and Hearing Research, 42, 1367-1377.

Haselager, G. J. T., Slis, I. H., \& Rietveld, A. C. M. (1991). An alternative method of studying the development of speech rate. Clinical Linguistics \& Phonetics, 5, 53-63.

Jeannerod, M. (1988). The neural and behavioural organization of goal-directed movements. Oxford: Oxford University Press.
Kelly, E. M., \& Conture, E. G. (1992). Speaking rates, response time, latencies, and interrupting behaviors of young stutterers, nonstutterers, and their mothers. Journal of Speech and Hearing Research, 35, 1256-1267.

Kowal, S., O'Connell, D. C., \& Sabin, E. J. (1975). Development of temporal patterning and vocal hesitations in spontaneous speech. Journal of Psycholinguistic Research, 4, 195-207.

Lindblom, B. (1990). Explaining phonetic variation: A sketch of the H\&H theory. In W. J. Hardcastle \& A. Marchal (eds.), Speech production and speech modelling (pp. 403-439). Netherlands: Kluwer Academic.

Mefferd, A. S., \& Green, J. R. (2010). Articulatory-to-acoustic relations in response to speaking rate and loudness manipulations. Journal of Speech, Language, and Hearing Research, 53, 1206-1219.

Mitchell, H. L., Hoit, J. D., \& Watson, P. J. (1996). Cognitivelinguistic demands and speech breathing. Journal of Speech and Hearing Research, 39, 93-104.

Müller, K., \& Hömberg, V. (1992). Development of speed of repetitive movements in children is determined by structural changes in corticospinal efferents. Neuroscience Letters, 144, 57-60.

Müller, K., Hömberg, V., \& Lenard, H. G. (1991). Magnetic stimulation of motor cortex and nerve roots in children: Maturation of cortico-motoneuronal projections. Electroencephalography and Clinical Neurophysiology, 8, 163-170.

Munson, B., Swenson, C. L., \& Manthei, S. C. (2005). Lexical and phonological organization in children: Evidence from repetition tasks. Journal of Speech, Language and Hearing Research, 48, 108-124.

Ostry, D. J., Keller, E., \& Parush, A. (1983). Similarities in the control of the speech articulators and the limbs: Kinematics of tongue dorsum movement in speech. Journal of Experimental Psychology. Human Perception and Performance, 9, 622-636.

Oytam, Y., Neilson, P. D., \& O’Dwyer, N. J. (2005). Degrees of freedom and motor planning in purposive movement. $\mathrm{Hu}$ man Movement Science, 24, 710-730.

Pindzola, R. H., Jenkins, M. M., \& Lokken, K. J. (1989). Speaking rates of young children. Language, Speech, and Hearing Services in Schools, 20, 133-138.

Potter, N. L., Kent, R. D., Lindstrom, M. J., \& Lazarus, J. C. (2006). Power and precision grip force control in three-tofive-year-old children: Velocity control precedes amplitude control in development. Experimental Brain Research, 172, 246-260.

Riely, R. R., \& Smith, A. (2003). Speech movements do not scale by orofacial structure size. Journal of Applied Physiology, 94, 2119-2126.

Rochester, S. R. (1973). The significance of pauses in spontaneous speech. Journal of Psycholinguistic Research, 2, 51-81.

Ryan, B. (2000). Speaking rate, conversational speech acts, interruption, and linguistic complexity of 20 pre-school stut- 
tering and non-stuttering children and their mothers. Clinical Linguistics \& Phonetics, 14, 25- 51.

Sadagopan, N., \& Smith, A. (2008). Developmental changes in the effects of utterance length and complexity on speech movement variability. Journal of Speech, Language and Hearing Research, 51, 1138-1151. doi: 10.1044/1092-4388(2008/06-0222)

Semel, E., Wiig, E. H., \& Secord, W. A. (2004). Clinical Evaluation of Language Fundamentals screening test (4th ed.). San Antonio, TX: Psychology Corporation.

Smith, A., \& Goffman, L. (1998). Stability and patterning of speech movement sequences in children and adults. Journal of Speech, Language and Hearing Research, 41, 18-30.

Smith, A., \& Zelaznik, H. N. (2004). Development of functional synergies for speech motor coordination in childhood and adolescence. Developmental Psychobiology, 45, 22-33.

Smith, B. L., \& Gartenberg, T. E. (1984). Initial observations concerning developmental characteristics of labiomandibular kinematics. Journal of Acoustic Society of America, 75, 1599-1605.

Stoel-Gammon, C. (2011). Relationships between lexical and phonological development in young children. Journal of Child Language, 38, 1-34. doi: 10.1017/ S0305000910000425

Sturm, J. A., \& Seery, C. H. (2007). Speech and articulatory rates of school-age children in conversation and narrative contexts. Language, Speech, and Hearing Services in Schools, 38, 47-59. doi: 10.1044/0161-1461(2007/005)
Vorperian, H. K., Wang, S., Chung, M. K., Schimek, E. M., Durtschi, R. B., Kent, R. D., et al. (2009). Anatomic development of the oral and pharyngeal portions of the vocal tract: An imaging study. Journal of the Acoustical Society of America, 125, 1666-1678. doi: 10.1121/1.3075589

Walker, J. F., \& Archibald, L. M. D. (2006). Articulation rate in preschool children: A 3-year longitudinal study. International Journal of Language $\mathcal{E}$ Communication Disorders, 41, 541-565.

Walker, J. F., Archibald, L. M. D., Cherniak, S. R., \& Fish, V. G. (1992). Articulation rate in 3- and 5-year-old children. Journal of Speech, Language and Hearing Research, 35, 4-13.

Walsh, B., \& Smith, A. (2002). Articulatory movements in adolescents: Evidence for protracted development of speech motor control processes. Journal of Speech, Language, and Hearing Research, 45, 1119-1133.

Westbury, J. R., \& Dembowski, J. (1993). Articulatory kinematics of normal diadochokinetic performance. Annual Bulletin-Research Institute of Logopedics and Phoniatrics, 27, 13-36.

Wiig, E. H., Secord, W. A., \& Semel, E. (2004). Clinical Evaluation of Language Fundamentals-Preschool (2nd ed.). San Antonio, TX: Psychology Corporation.

Ziegler, W. (2002). Task-related factors in oral motor control: Speech and oral diadochokinesis in dysarthria and apraxia of speech. Brain and Language, 80, 556-575. doi: 10.1006/ brln.2001.2614 